\title{
An Exact Solution of the Binary Singular Problem
}

\author{
Baiqing Sun, Kun Tang, Hongmei Zhang, and Shan Xiong
}

School of Management, Harbin Institute of Technology, 150001 Harbin, China

Correspondence should be addressed to Baiqing Sun; baiqingsun@hit.edu.cn and Hongmei Zhang; zhanghongmei223@163.com

Received 11 November 2013; Accepted 21 January 2014; Published 9 April 2014

Academic Editor: Donghai Ji

Copyright (C) 2014 Baiqing Sun et al. This is an open access article distributed under the Creative Commons Attribution License, which permits unrestricted use, distribution, and reproduction in any medium, provided the original work is properly cited.

\begin{abstract}
Singularity problem exists in various branches of applied mathematics. Such ordinary differential equations accompany singular coefficients. In this paper, by using the properties of reproducing kernel, the exact solution expressions of dual singular problem are given in the reproducing kernel space and studied, also for a class of singular problem. For the binary equation of singular points, I put it into the singular problem first, and then reuse some excellent properties which are applied to solve the method of solving differential equations for its exact solution expression of binary singular integral equation in reproducing kernel space, and then obtain its approximate solution through the evaluation of exact solutions. Numerical examples will show the effectiveness of this method.
\end{abstract}

\section{Introduction}

Singular problems exist in various branches of applied mathematics. The coefficients of one or some of the items in a given interval are of no significance at some point. It is difficult to solve the singular boundary value problems in the numerical calculation, and the classical numerical methods are not able to get a better numerical approximation. Based on the reproducing kernel space, it gives an extract solution expression by using the properties of reproducing kernel.

Generally, reproducing kernel theory can be divided into two aspects. On the one hand produced in integral theory, then the nuclear is considered as the definite integral operator continuous kernel. This theory is initiated by Mercer [1] in the term of "positive definite kernel," which is equated by other scholars interested in references in the 1920s. Mercer found that continuous nuclear of positive definite integral equation has the following properties:

$$
\sum_{i, j=1}^{n} K\left(y_{i}, y_{j}\right) \xi_{i} \xi_{j} \geq 0
$$

In the 1930s, E. H. Moore also found the same nature. He discussed the kernel function $K(x, y)$ which is defined in the abstract set $E$ with property (1) and is used in the generalized integral equation in the analysis of "definite Hermitian Matrix" term. He proved that for every positive definite Hermitian matrix corresponding to a family of functions forms the Hilbert space which has the inner product $(f, g)$, and the nuclear in this space has the property of reproducibility

$$
f(y)=(f(x), K(x, y)) .
$$

Such a discovery connects two kinds of views of reproducing kernel, and this theory has also been proposed by Bochner in the term of "positive definite function" [2].

On the other hand the reproducing kernel theory produced in the article on harmonic boundary value of the biharmonic function written by Zaremba. Zaremba [3] was the first one who introduced a nuclear corresponding to a family of functions in special cases and proved the reproducibility (2). Aronszajn summarized previous work in 1943 and formed the theory of reproducing kernel, including the formation of a special case of the Bergman kernel function of the system [4]. In 1970, Larkin [5] solved the problem of the optimal approximation rules of nuclear regeneration in the Hilbert function space. While Chawla and Kaul gave the other optimal approximation rules [6] with polynomial precision in the Hilbert function space in 1974. Since then, a large number of foreign scholars discussed the reproducing kernel problems [7], done a lot of research work [8], and created many of the reproducing kernel construction methods [9] and how to make use of the kernel function of regeneration 
solving equations. Chacaltana et al. [10] introduced quantum Hilbert space innovatively and set up the reproducing kernel in this space.

The domestic research in the field of reproducing kernel and its properties began in the 1980s, Cui et al. [11, 12]. His main study is reproducing kernel space approximation theory and numerical methods. He constructed a reproducing kernel space $W_{2}^{1}[a, b]$ and its finite expressions and proved it is a reproducing kernel Hilbert space. In 1986, he made a more in-depth research in the space $W_{2}^{1}[a, b]$ [13] and obtained the optimal interpolation approximation expression. Thereafter, Várady et al. [14] discussed the surface interpolation together in the space. Kineri et al. [15] gave his definition of reproducing kernel space $W_{2}^{1}(D)$ in twodimensional rectangular area $D=[a, b] \times[c, d] \subset R^{2}$. Jordan studied multivariate interpolation in the space $W_{2}^{1}(D)$ [16], and gave a multivariate interpolation formula. That same year, she further addressed the problem of computing multivariate interpolation. Fasshauer and Ye [17] studied the best approximation of bounded linear operator in $W$ space. Boying [18] discussed the spline interpolation of differential operator in $W_{2}^{1}$. Meanwhile, Cui also defines some of the other reproducing kernel space in which approximate linear operator equations is studied to solve the problem. In addition, $\mathrm{Wu}$ and Zhang [19] give the reproducing kernel space, and the operator equation was solved in this space. Mohammadi and Mokhtari also put forward a reproducing kernel space in the literature [20], obtained the expression of reproducing kernel by convolution, and presented the expressions of analytical solution and numerical solution of a high order partial differential equation. Arqub et al. [21] give the accurate solution of a class of integrodifferential equations in reproducing kernel space. In recent years, people began to discuss numerical solution of nonlinear operator equation in the reproducing kernel space. Wang et al. [22] discussed approximate nonlinear operator equation solving problems in the reproducing kernel space; the equation is

$$
A u B u+C u=f .
$$

Here $A, B, C: W_{2}^{1} \rightarrow W_{2}^{1}$ are bounded linear operator. Eigenvalue method and the factorization method which are obtained by using the good nature of reproducing kernel are the two solutions of (3).

Reproducing kernel space gives the ideal space framework of numerical analysis problem; in this space there is a function which makes the function in the corresponding space demonstrate the reproducibility through the inner product, so for the numerical analysis of the basic value of operation, there is a continuous signal. This is why the domestic and foreign scholars have devoted much energy to study the theory of reproducing kernel. Precisely because the problem of discrete values can be continuously shown, various types of optimization of numerical problems become possible. In addition, the regeneration of nuclear technique, combined with other direction, has produced many new theories and algorithms, such as signal processing, stochastic processes processing, estimation theory, wavelet transform [23], reproducing kernel particle method, and others which have many improvement application examples. Obviously, such functional analysis tools have good qualities whether in establishing theoretical framework or in a numerical algorithm.

In this paper, first of all, transform the dual singular equation into nonsingular problems, and then use the method of solving differential equations to solve the nonsingular problem by using some excellent properties in reproducing kernel space, resulting in exact solution expression of binary singular equation, finally get the approximate solution through the exact solution evaluation. At the same time, the paper calculates the error of the approximate solution through numerical example; it turns out the proposed method has the feasibility and effectiveness.

\section{Theory Review}

\subsection{The Definition of Reproducing Kernel Space $W_{2}^{1}[a, b]$ and Reproducing Kernel Method}

2.1.1. Definition of Reproducing Kernel Space $W_{2}^{1}[a, b]$. $W_{2}^{1}[a, b]=\{u(x) \mid u(x)$ is a unary real absolutely continuous function confined to $[a, b]$, besides $\left.u^{\prime}(x) \in L^{2}[a, b]\right\}$. For any $u(x), v(x) \in W_{2}^{1}[a, b]$, define the inner product and norm as follows:

$$
\begin{gathered}
(u(x), v(x))_{W_{2}^{1}}=\int_{a}^{b}\left(u(x) v(x)+u^{\prime}(x) v^{\prime}(x)\right) d x, \\
\|u\|=(u, u)_{W_{2}^{1}}^{1 / 2} .
\end{gathered}
$$

For details about reproducing kernel space, see [23]; the space $W_{2}^{1}[a, b]$ on the norm (5) is a complete inner product space.

2.1.2. The Reproducing Kernel Solution of $W_{2}^{1}[0,1]$. Look at the function

$$
(f, g)=\int_{0}^{1} f g+f^{\prime} g^{\prime} d x=\left.f g^{\prime}\right|_{0} ^{1}+\int_{0}^{1} f\left(g-g^{\prime \prime}\right) d x
$$

Let

$$
\delta(y)=R_{x}(y)
$$

Then get

$$
\begin{aligned}
\left(f(y), R_{x}(y)\right)= & \left.f(y) R_{x}^{\prime}(y)\right|_{0} ^{1} \\
& +\int_{0}^{1} f(y)\left(R_{x}(y)-R_{x}^{\prime \prime}(y)\right) d y .
\end{aligned}
$$

In order to make

$$
\left(f(y), R_{x}(y)\right)=f(x)
$$

only make

$$
\begin{aligned}
R_{x}(y)-R_{x}^{\prime \prime}(y) & =\delta(y-x), \\
R_{x}^{\prime}(1) & =0, \\
R_{x}^{\prime}(0) & =0
\end{aligned}
$$


each formula of the above equations, respectively, denoted as: (10), (11), and (12).

By (10), when $y \neq x$, there is $R_{x}(y)-R_{x}^{\prime \prime}(y)=0$; the characteristic equation is $1-\lambda^{2}=0$, then $\lambda= \pm 1$.

So

$$
R_{x}(y)= \begin{cases}c_{1}(x) e^{y}+c_{2}(x) e^{-y}, & y \leq x, \\ d_{1}(x) e^{y}+d_{2}(x) e^{-y}, & y>x\end{cases}
$$

For (10), integral to

$$
\begin{gathered}
\int_{x-\varepsilon}^{x+\varepsilon} R_{x}(y)-R_{x}^{\prime \prime}(y) d y=\int_{x-\varepsilon}^{x+\varepsilon} \delta(y-x) d y=1 \\
\left.u(1)\left(R_{x}(y)\right)_{y}^{\prime \prime}\right|_{y=1}-\left.u(0)\left(R_{x}(y)\right)_{y}^{\prime \prime}\right|_{y=0} \\
-\left.\left(R_{x}(y)\right)_{y}^{\prime}\right|_{x-\varepsilon} ^{x+\varepsilon}=1 .
\end{gathered}
$$

Let $\varepsilon \rightarrow 0$, then get

$$
\begin{gathered}
R_{x}(x+0)=R_{x}(x-0), \\
R_{x}^{\prime}(x-0)-R_{x}^{\prime}(x+0)=1 .
\end{gathered}
$$

The two formulas of the above equations, respectively, denoted by (15) from (11), (12), (15) we can get

$$
R_{x}(y)=\left\{\begin{array}{l}
\frac{e^{x+y}+e^{2-(x+y)} e^{x-y}+e^{2+y-x}}{2\left(e^{2}-1\right)} \\
\frac{e^{x+y}+e^{2+(x+y)} e^{y-x}+e^{2+x-y}}{2\left(e^{2}-1\right)} .
\end{array}\right.
$$

2.2. The Definition and Basic Properties of Reproducing Kernel Space $W_{2,0}^{3}$. For different equation, we will construct different reproducing kernel space according to the actual situation. The literature [23] has given some reproducing kernel space. Although these spaces have different definition, inner product and reproducing kernel function, their corresponding reproducing kernel function has the regeneration properties similar to (10). Take the reproducing kernel space $W_{2,0}^{3}$ for example; give its method of construction and its algorithm. follows:

The definition of the reproducing kernel space $W_{2,0}^{3}$ is as

$$
W_{2,0}^{3}[a, b]=u(x) .
$$

Here $u(x), u^{\prime}(x), u^{\prime \prime}(x)$ is a unary real absolutely continuous function affiliated to $[a, b], u^{\prime \prime \prime}(x) \in L^{2}[a, b], u(a)=$ $u(b)=0$.

For any $u(x), v(x) \in W_{2,0}^{3}[a, b]$, its inner product and norm are defined as follows:

$$
\begin{gathered}
(u(x), v(x))_{W_{2}^{3}}=\int_{a}^{b}\left(u(x) v(x)+3 u^{\prime}(x) v^{\prime}(x)\right. \\
\left.+3 u^{\prime \prime}(x) v^{\prime \prime}(x)+u^{\prime \prime \prime}(x) v^{\prime \prime \prime}(x)\right) d x \\
\|u\|=(u, u)_{W_{2,0}^{3}}^{1 / 2} .
\end{gathered}
$$

Similar to literature [23], we can prove that the reproducing kernel space $W_{2}^{3}$ on norm (19) is a Hilbert space.

The algorithm

$$
\begin{aligned}
& \left(u(t), R_{x}(t)\right) \\
& =\int_{a}^{b} u(t) R_{x}(t)+3 u^{\prime}(t) R_{x}^{\prime}(t) \\
& +3 u^{\prime \prime}(t) R_{x}^{\prime \prime}(t)+u^{\prime \prime \prime}(t) R_{x}^{\prime \prime \prime}(t) d t \\
& \int_{a}^{b} 3 u^{\prime}(t) R_{x}^{\prime}(t) d t=-3 \int_{z}^{b} R_{x}^{\prime \prime}(t) u(t) d t+\left.3 u(t) R_{x}^{\prime}(t)\right|_{a} ^{b} \\
& \int_{a}^{b} 3 u^{\prime \prime}(t) R_{x}^{\prime \prime}(t) d t \\
& =\left.3 u^{\prime}(t) R_{x}^{\prime \prime}(t)\right|_{a} ^{b}-3 \int_{a}^{b} u^{\prime}(t) R_{x}^{\prime \prime \prime}(t) \\
& =\left.3 u^{\prime}(t) R_{x}^{\prime \prime}(t)\right|_{a} ^{b}-\left.3 u(t) R_{x}^{\prime \prime \prime}\right|_{a} ^{b}+3 \int_{a}^{b} u(t) R_{x}^{\prime \prime \prime}(t) d t \\
& \int_{a}^{b} u^{\prime \prime \prime}(t) R_{x}^{\prime \prime \prime}(t) d t \\
& =\left.u^{\prime \prime}(t) R_{x}^{\prime \prime \prime}(t)\right|_{a} ^{b}-\int_{a}^{b} u^{\prime \prime}(t) R_{x}^{(4)}(t) d t \\
& =\left.u^{\prime \prime}(t) R_{x}^{\prime \prime \prime}(t)\right|_{a} ^{b}-\left.u^{\prime}(t) R_{x}^{(4)}(t)\right|_{a} ^{b} \\
& +\int_{a}^{b} u^{\prime}(t) R_{x}^{(5)}(t) d t \\
& =\left.u^{\prime \prime}(t) R_{x}^{\prime \prime \prime}(t)\right|_{a} ^{b} \\
& -\left.u^{\prime}(t) R_{x}^{(4)}(t)\right|_{a} ^{b}+\left.u(t) R_{x}^{(5)}(t)\right|_{a} ^{b} \\
& -\int_{a}^{b} u(t) R_{x}^{(6)}(t) d t .
\end{aligned}
$$

Tidy is as follows:

$$
\begin{aligned}
(u(t), & \left.R_{x}(t)\right) \\
= & \int_{a}^{b} u(t)\left[R_{x}(t)-3 R_{x}^{\prime \prime}(t)+3 R_{x}^{(4)}(t)-R_{x}^{(6)}(t)\right] d t \\
& +\left.u(t)\left[3 R_{x}^{\prime}(t)-3 R_{x}^{(3)}(t)+R_{x}^{(5)}(t)\right]\right|_{a} ^{b} \\
& +\left.u^{\prime}(t)\left[3 R_{x}^{\prime \prime}(t)-R_{x}^{(4)}(t)\right]\right|_{a} ^{b}+\left.u^{\prime \prime}(t) R_{x}^{(3)}(t)\right|_{a} ^{b}
\end{aligned}
$$


In order to make $\left(u(t), R_{x}(t)\right)=u(x)$, just let

$$
\begin{gathered}
R_{x}(t)-3 R_{x}^{\prime \prime}(t)+3 R_{x}^{(4)}(t)-R_{x}^{(6)}(t)=\delta(x-t), \\
\left.u(t)\left[3 R_{x}^{\prime}(t)-3 R_{x}^{(3)}(t)+R_{x}^{(5)}(t)\right]\right|_{a} ^{b}=0, \\
\left.u^{\prime}(t)\left[3 R_{x}^{\prime \prime}(t)-R_{x}^{(4)}(t)\right]\right|_{a} ^{b}=0, \\
\left.u^{\prime \prime}(t) R_{x}^{(3)}(t)\right|_{a} ^{b}=0 .
\end{gathered}
$$

By (22), when $x \neq t, \delta(x-t)=0$, the characteristic equation is $1-3 \lambda^{2}+3 \lambda^{4}-\lambda^{6}=0$, so

$$
R_{x}=\left\{\begin{array}{rr}
\left(c_{1}+c_{2} y+c_{3} y^{2}\right) e^{y} & \\
+\left(c_{4}+c_{5} y+c_{6} y^{2}\right) e^{-y}, & y \leq x \\
\left(d_{1}+d_{2} y+d_{3} y^{2}\right) e^{y} & \\
+\left(d_{4}+d_{5} y+d_{6} y^{2}\right) e^{-y}, & y>x
\end{array}\right.
$$

By (22) to know, when $k=4,\left(R_{x}(y)\right)_{y}^{(k)}$ is continues, when $\left(R_{x}(y)\right)_{y}^{(5)}, x=y$, there is a Jump $(-1)^{3-1}=1$, so $R_{x}(y)$ satisfies the following conditions:

$$
\begin{gathered}
\left(R_{x}(x-0)\right)_{t}^{(k)}=\left(R_{x}(x+0)\right)_{t}^{(k)}, \quad k=0,1,2,3,4, \\
\left(R_{x}(x-0)\right)_{t}^{(5)}-\left(R_{x}(x+0)\right)_{t}^{(5)}=1 .
\end{gathered}
$$

Put (23) into concrete expansion:

$$
\begin{aligned}
\left.u(t)\left[3 R_{x}^{\prime}(t)-3 R_{x}^{(3)}(t)+R_{x}^{(5)}(t)\right]\right|_{a} ^{b} & \\
& +\left.u^{\prime}(t)\left[3 R_{x}^{\prime \prime}(t)-R_{x}^{(4)}(t)\right]\right|_{a} ^{b}+\left.u^{\prime \prime}(t) R_{x}^{(3)}(t)\right|_{a} ^{b}=0 .
\end{aligned}
$$

Further to know

$$
\begin{gathered}
R_{x}(a)=0, \\
R_{x}(b)=0, \\
{\left.\left[3 R_{x}^{\prime \prime}(t)-R_{x}^{(4)}(t)\right]\right|_{a}=0,} \\
{\left.\left[3 R_{x}^{\prime \prime}(t)-R_{x}^{(4)}(t)\right]\right|_{b}=0,} \\
\left.R_{x}^{(3)}(t)\right|_{a}=0, \\
\left.R_{x}^{(3)}(t)\right|_{b}=0 .
\end{gathered}
$$

From (27) to (35), a total of 8 equations, we can use a mathematic software programming to work out $R_{x}(t) . R_{x}(t)$ can be applied to reproducing kernel space $W_{2,0}^{3}[0,1]$, and then obtain the kernel function $R_{x}(y)$; its expression is

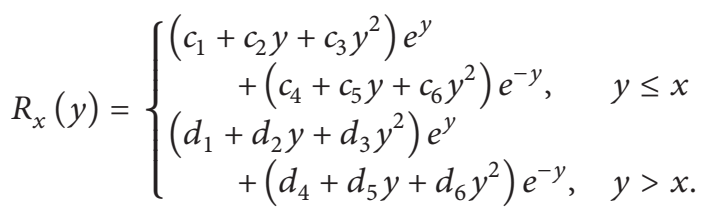

2.3. The Definition of $W_{1}(\Omega)$ and Its Reproducing Kernel Expression. The definition of $W_{1}(\Omega)$ is

$$
W_{1}(\Omega)=W_{2,0}^{3}[0,1] \times W_{2,0}^{3}[0,1]=u(x, y) .
$$

Here $u_{x^{p} y^{q}}$ is second order completely continuous on $D$ and $p, q=0,1,2, u(x, 0)=u(x, 1)=u(1, y)=0, u_{x^{p} y^{q}} \in$ $L^{2}(D), p, q=0,1,2,3$.

Its inner product is

$$
\begin{array}{rl}
(u, v)=\int_{D} & u v+3 u_{y} v_{y}+3 u_{y y} v_{y y}+u_{y y y} v_{y y y} \\
& +3 u_{x} v_{x}+9 u_{x x} v_{x x}+9 u_{x y y} v_{x y y}+3 u_{x y y y} v_{x y y y} \\
& +3 u_{x x} v_{x x}+9 u_{x x y} v_{x x y}+9 u_{x x y y} v_{x x y y} \\
& +3 u_{x x y y y} v_{x x y y y}+u_{x x x} v_{x x x}+3 u_{x x x y} v_{x x x y} \\
& +3 u_{x x x y y} v_{x x x y y}+u_{x x x y y y} v_{x x x y y y} d x d y .
\end{array}
$$

The norm is $\|u\|\langle u, u\rangle_{W_{1}(\Omega)}^{1 / 2}$.

The reproducing kernel before and after the space $W_{1}(\Omega)=W_{2,0}^{3}[0,1] \times W_{2,0}^{3}[0,1]$, respectively is $R_{\xi}^{1}(x)$ and $R_{\eta}^{2}(y)$.

Proof. $R_{\xi}^{1}(x) R_{\eta}^{2}(y)$ is the reproducing kernel of $W_{1}(\Omega)$.

The proof of renewable: for all $u(x, y) \in W(\Omega)$

$$
\begin{aligned}
&(u(x, y),\left.R_{\varepsilon}^{1}(x) R_{\eta}^{2}(y)\right)_{W_{1}(\Omega)} \\
&=\iint_{0}^{1} u(x, y) R_{\varepsilon}^{1}(x) R_{\eta}^{2}(y)+3 u_{y} R_{\varepsilon}^{1}(x)\left(R_{\eta}^{2}(y)\right)_{y} \\
&+3 u_{y y} R_{\varepsilon}^{1}(x)\left(R_{\eta}^{2}(y)\right)_{y y} \\
&+u_{y y y} R_{\varepsilon}^{1}(x)\left(R_{\eta}^{2}(y)\right)_{y y y} \\
&+3 u_{x}\left(R_{\xi}^{1}(x)\right)_{x}^{\prime} R_{\eta}^{2}(y) \\
&+9 u_{x y}\left(R_{\xi}^{1}(x)\right)_{x}\left(R_{\eta}^{2}(y)\right)_{y} \\
&+9 u_{x y y}\left(R_{\xi}^{1}(x)\right)_{x}\left(R_{\eta}^{2}(y)\right)_{y y} \\
&+3 u_{x y y y}\left(R_{\xi}^{1}(x)\right)_{x}\left(R_{\eta}^{2}(y)\right)_{y y y} \\
&+3 u_{x x}\left(R_{\xi}^{1}(x)\right)_{x x} R_{\eta}^{2}(y) \\
&+9 u_{x x y}\left(R_{\xi}^{1}(x)\right)_{x x}\left(R_{\eta}^{2}(y)\right)_{y} \\
&+9 u_{x x y y}\left(R_{\xi}^{1}(x)\right)_{x x}\left(R_{\eta}^{2}(y)\right)_{y y} \\
&+3 u_{x x y y y}\left(R_{\xi}^{1}(x)\right)_{x x}\left(R_{\eta}^{2}(y)\right)_{y y y} \\
&+3 u_{x x x}\left(R_{\xi}^{1}(x)\right)_{x x x} R_{\eta}^{2}(y)
\end{aligned}
$$




$$
\begin{aligned}
& +9 u_{x x x y}\left(R_{\xi}^{1}(x)\right)_{x x x}\left(R_{\eta}^{2}(y)\right)_{y} \\
& +9 u_{x x x y y}\left(R_{\xi}^{1}(x)\right)_{x x x}\left(R_{\eta}^{2}(y)\right)_{y y} \\
& +3 u_{x x x y y y}\left(R_{\xi}^{1}(x)\right)_{x x x}\left(R_{\eta}^{2}(y)\right)_{y y y} d x d y \\
= & \int_{0}^{1} R_{\eta}^{2}(y)\left(u(x, y), R_{\xi}^{1}(x)\right) d y \\
+ & 3 \int_{0}^{1}\left(R_{\eta}^{2}(y)\right)_{y}\left(u_{y}, R_{\xi}^{1}(x)\right) d y \\
& +3 \int_{0}^{1}\left(R_{\eta}^{2}(y)\right)_{y y}\left(u_{y y}, R_{\xi}^{1}(x)\right) d y \\
& +\int_{0}^{1}\left(R_{\eta}^{2}(y)\right)_{y y y}\left(u_{y y y}, R_{\xi}^{1}(x)\right) d y \\
= & \int_{0}^{1} u(\xi, y)\left(R_{\eta}^{2}(y)\right)+3 u_{y}(\xi, y)\left(R_{\eta}^{2}(y)\right)_{y} \\
& +3 u_{y y}(\xi, y)\left(R_{\eta}^{2}(y)\right)_{y y} \\
& +u_{y y y}(\xi, y)\left(R_{\eta}^{2}(y)\right)_{y y y} d y \\
= & \left(u(\xi, y), R_{\eta}^{2}(y)\right)_{y}=u(\xi, \eta) .
\end{aligned}
$$

Fix $\xi, \eta$, then $R_{\xi}^{1}(y)$ and $R_{\eta}^{2}(y)$ are absolutely continuous on $x$, $y$.

Proof $\partial^{p+q}\left(R_{\xi}^{1}(x) R_{\eta}^{2}(y)\right) / \partial x^{p} \partial y^{q}$ is absolutely continuous, while $R_{\xi}^{1}(x) R_{\eta}^{2}(y)$ is second order completely continuous on $(x, y)$. And the following are established:

$$
\begin{gathered}
\left(R_{\xi}^{1}(x)\right)_{x} R_{\eta}^{2}(y) \in L^{2}(D) R_{\xi}^{1}(x)\left(R_{\eta}^{2}(y)\right)_{y} \in L^{2}(D), \\
\left(R_{\xi}^{1}(x)\right)_{x}\left(R_{\eta}^{2}(y)\right)_{y} \in L^{2}(D) R_{\xi}^{1}(x) R_{\eta}^{2}(y) \in W_{1}(\Omega) .
\end{gathered}
$$

So the reproducing kernel may consist of the product of two Spaces.

The results obtained in Section 2.3 can be moved over to get the reproducing kernel $R=R_{\xi}^{1}(x) R_{\eta}^{2}(y)$ of $W_{1}(\Omega)$.

\section{Solution of the Dual Singular Equations}

In this chapter, we will discuss the following singular equation in reproducing kernel space

$$
\begin{aligned}
-\frac{1}{p(x)} \frac{\partial}{\partial x}\left(p(x) a(x, y) u_{x}\right) & +\frac{\partial}{\partial y}\left(a(x, y) u_{y}\right)=f(x, y), \\
\left.u\right|_{\Gamma_{0}} & =0 .
\end{aligned}
$$

Here, $\Gamma_{0}$ is the periphery of $\Omega=[0,1] \times[0,1], P(0)=0$. Under the assumption that (41) has a unique solution, we will give its exact solution representation and approximate solution.
3.1. The Transformation of the Problem. Equation (41) can be converted into the solution of the following equation:

$$
\begin{aligned}
& -[p(x) a(x, y)]_{x}^{\prime} u_{x}-p(x) a(x, y) u_{x x}^{\prime \prime} \\
& \quad+p(x) a_{y}^{\prime}(x, y) u_{y}+p(x) a(x, y) u_{y y}^{\prime \prime} \\
& =p(x) f(x, y) .
\end{aligned}
$$

3.2. Space Election. The selection of reproducing kernel space is as follows.

The left choice is

$$
W_{1}(\Omega)=W_{2,0}^{3}[0,1] \times W_{2,0}^{3}[0,1] .
$$

The right choice is

$$
W(\Omega)=W_{2}^{1}[0,1] \times W_{2}^{1}[0,1] .
$$

Define the operator

$$
\begin{aligned}
L(u)(x)= & -[p(x) a(x, y)]_{x}^{\prime} u_{x}-p(x) a(x, y) u_{x x}^{\prime \prime} \\
& +p(x) a_{y}^{\prime}(x, y) u_{y}^{\prime}+p(x) a(x, y) u_{y y}^{\prime \prime} .
\end{aligned}
$$

Denoted as $L u=L_{1} u+L_{2} u+L_{3} u+L_{4} u$, among them:

$$
\begin{gathered}
L_{1} u=-[p(x) a(x, y)]_{x}^{\prime} u_{x} L_{2} u=-p(x) a(x, y) u_{x x}^{\prime \prime}, \\
L_{3} u=p(x) a_{y}^{\prime}(x, y) u_{y}^{\prime} L_{4} u=p(x) a(x, y) u_{y y}^{\prime \prime} .
\end{gathered}
$$

The following is the proof of $L$ boundedness.

Proved $L: W_{2,0}^{3}[0,1] \times W_{2,0}^{3}[0,1] \rightarrow W_{2}^{0}[0,1]$ is bounded linear operator

Proved $L_{1}: W_{1}(\Omega) \rightarrow W(\Omega)$ is bounded linear operator

(1) First Proof. For all $u \in W_{1}(\Omega)$, then $L_{1} u \in W(\Omega)$

Let $q(x, y)=-[p(x) a(x, y)]_{x}^{\prime}$. In fact, $q(x, y)$ and $u_{x}^{\prime}$ are completely continuous so $L_{1} u$ is completely continuous; furthermore $\left(L_{1} u\right)_{x}^{\prime}=q_{x}^{\prime}(x, y) u_{x}^{\prime}+q(x, y) u_{x x}^{\prime \prime}$.

Since $q_{x}^{\prime}(x, y), u_{x}^{\prime}, u_{x x}^{\prime \prime}$, and $q(x, y)$ are all continuous.

So $q_{x}^{\prime}(x, y) u_{x}^{\prime} \in L^{2}(\Omega)$ and $q(x, y) u_{x x}^{\prime \prime} \in L^{2}(\Omega)$ and yield $\left(L_{1} u\right)_{x}^{\prime} \in L^{2}(\Omega)$.

Similarly $\left(L_{1} u\right)_{y}^{\prime} \in L^{2}(\Omega)$.

And because $\left(L_{1} u\right)_{x y}^{\prime \prime}=q_{x y}^{\prime \prime}(x, y) u_{x}+q_{x}(x, y) u_{x y}+$ $q_{x}^{\prime}(x, y) u_{x x}+q_{x}(x, y) u_{x x y}$.

We can get $\left(L_{1} u\right)_{x y}^{\prime \prime} \in L^{2}(\Omega)$ from the continuous $q_{x y}^{\prime \prime}(x, y), u_{x x y} \in L^{2}(\Omega)$.

Therefore, $L_{1} u \in W(\Omega)$.

(2) The Linearity of $L_{1} u$ Is Obvious. Next we prove $L_{1}$ : $W_{1}(\Omega) \rightarrow W(\Omega)$ is bounded. Namely, proof is as follows: for all $u \in W_{1}(\Omega)$

$$
\exists M>0, \quad \text { make }\left\|L_{1} u\right\|_{W(\Omega)} \leq M\|u\|_{W_{1}(\Omega)} .
$$


Actually,

$$
\begin{gathered}
\left\|L_{1} u\right\|_{W(\Omega)}^{2}=\int_{\Omega}\left(L_{1} u\right)^{2}+\left(L_{1} u\right)_{x}^{2}+\left(L_{1} u\right)_{y}^{2}+\left(L_{1} u\right)_{x y}^{2} d x d y \\
\int_{\Omega}\left(L_{1} u\right)^{2} d x d y=\int_{\Omega} q^{2}(x, y) u_{x}^{2} d x d y \\
\leq M \int_{\Omega} u_{x}^{2} d x d y \leq M\|u\|_{W_{1}(\Omega)}^{2}, \\
\int_{\Omega}\left(L_{1} u\right)_{x}^{2} d x d y=\int_{\Omega}\left(q_{x}^{\prime}(x, y) u_{x}^{\prime}+q(x, y) u_{x x}^{\prime \prime}\right)^{2} d x d y \\
=\int_{\Omega}\left(q_{x}^{\prime}(x, y) u_{x}^{\prime}\right)^{2}+2 q_{x}^{\prime}(x, y) \\
\times u_{x}^{\prime} q(x, y) u_{x x}^{\prime \prime}+\left(q(x, y) u_{x x}^{\prime \prime}\right)^{2} d x d y \\
\leq M\|u\|_{W_{1}(\Omega)}^{2},
\end{gathered}
$$$$
\int_{\Omega}\left(L_{1} u\right)_{y}^{2} d x d y \leq M\|u\|_{W_{1}(\Omega)}^{2}
$$$$
\int_{\Omega}\left(L_{1} u\right)_{x y}^{2} d x d y \leq M\left\|u u_{x}\right\|_{W_{1}(\Omega)}^{2} .
$$

Similarly to prove $L_{2} u=-p(x) a(x, y) u_{x x}^{\prime \prime}, L_{3} u=$ $p(x) a_{y}^{\prime}(x, y) u_{y}^{\prime}, L_{4} u=p(x) a(x, y) u_{y y}^{\prime \prime}$ are linear bounded.

3.3. Expression of the Solution. The solution of $L_{1} u$ : $W_{1}(\Omega) \rightarrow W(\Omega)$ is unique.

Assume $\left\{M_{i}\right\}_{i=1}^{\infty}$ belongs to $\Omega$; note $\varphi_{i}(M)=R_{M_{i}}(M)$, $\Psi_{i}(M)=\left(L^{*} \varphi_{i}\right)(M), \bar{\Psi}_{i}=\sum_{k=1}^{i} \beta_{i k} \Psi_{k}$.

Theorem 1. $\left\{\Psi_{i}\right\}_{i=1}^{\infty}$ is perfect in $W_{1}(\Omega)$.

Proof. For all $u \in W_{1}(D)$ satisfy $\left(u, \Psi_{i}\right)=0$.

The Certificate. $u=0$. In fact, $0=\left(u, \psi_{i}\right)=\left(u, L^{*} \varphi_{i}\right)=$ $\left(L u, \varphi_{i}\right)=(L u)_{M_{i}}$.

So $(L u)_{(M)}=0$ and since $L$ is injective, therefore $u=0$.

Theorem 2. The true solution of $L u=f$ is

$$
u(M)=\sum_{i=1}^{\infty}\left[\sum_{k=1}^{i} \beta_{i k} f\left(M_{k}\right)\right] \bar{\psi}_{i}
$$

Prove

$$
\begin{aligned}
\left(u, \bar{\psi}_{i}\right) & =\sum_{k=1}^{i} \beta_{i k}\left(u, \psi_{k}\right)=\sum_{k=1}^{i} \beta_{i k}\left(u, L^{*} \psi_{k}\right) \\
& =\sum_{k=1}^{i} \beta_{i k}\left(L u, \psi_{k}\right)=\sum_{k=1}^{i} \beta_{i k}\left(f, \psi_{k}\right)=\sum_{k=1}^{i} \beta_{i k} f\left(M_{k}\right) .
\end{aligned}
$$

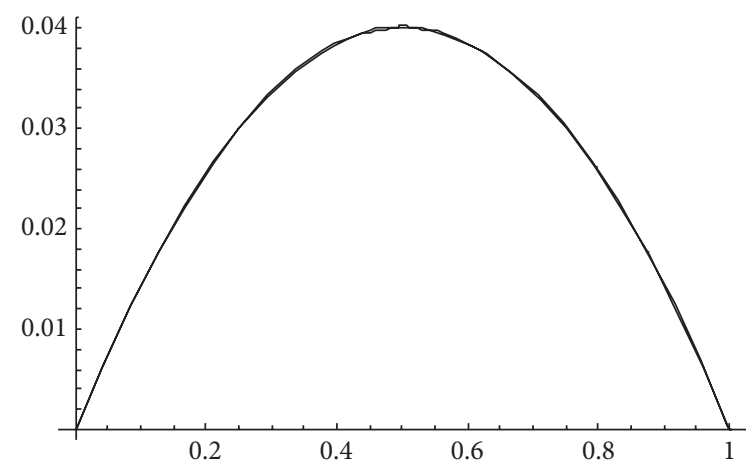

FIGURE 1: $y=0.2$, the approximate solution $u(x)$, the exact solution $\operatorname{utrue}(x), x \in[0,1]$.

The exact solution is $u=\sum_{i=1}^{\infty}\left(u, \bar{\psi}_{i}\right) \bar{\psi}_{i}=$ $\sum_{i=1}^{\infty}\left[\sum_{k=1}^{i} \beta_{i k} f\left(M_{k}\right)\right] \bar{\psi}_{i}$.

The approximate solution is $u_{n}=\sum_{i=1}^{n}\left(u, \bar{\psi}_{i}\right) \bar{\psi}_{i}=$ $\sum_{i=1}^{n}\left[\sum_{k=1}^{i} \beta_{i k} f\left(M_{k}\right)\right] \bar{\psi}_{i}$.

Then get the exact solution for $n$ entry truncation in order to, respectively, get the approximate solution expression $u_{n}(x)$ on $[0,1]$.

\section{Numerical Example Analysis}

In this chapter we will use the previously mentioned methods to calculate a numerical example and use it to test the validity of the application; all operations are running under the environment of Mathematica 5.0 mathematical software. From the numerical results it can be seen that our method is very effective.

Example 3. Solve the following dual singular problem

$$
\begin{gathered}
-\frac{1}{x} \frac{\partial}{\partial x}\left(x \cdot x y \cdot u_{x}^{\prime}(x, y)\right)+\frac{\partial}{\partial y}\left(x y \cdot u_{y}^{\prime}(x, y)\right)=f(x, y) \\
\left.u\right|_{\Gamma_{0}}=0 .
\end{gathered}
$$

Here $\Gamma_{0}$ is the boundary of $\Omega=[0,1] \times[0,1]$. Exact solutions for the given equation are $u(x)=x(1-x) y(1-y)$; we select 36 points in the rectangular area and, respectively, let $y=0.2, y=0.9$. The graphics of exact solution utrue $(x)$ and approximate solution $u(x)$ are shown in Figures 1 and 3. In Figure 1, $y=0.2$; the curve of approximate solution is almost the same as that of the exact solution. In Figure 3, $y=0.9$, we can tell the difference between the curve of the approximate solution $u(x)$ and the exact solution $u$ true $(x), x \in[0,1]$. Figure 2 shows the absolute error of the approximate solution $u(x)$ and the exact solution utrue $(x), x \in[0,1]$, when $y=0.2$. And when $y=0.9$. Figure 4 indicates the absolute error of the approximate solution $u(x)$ and the exact solution utrue $(x), x \in[0,1]$. 


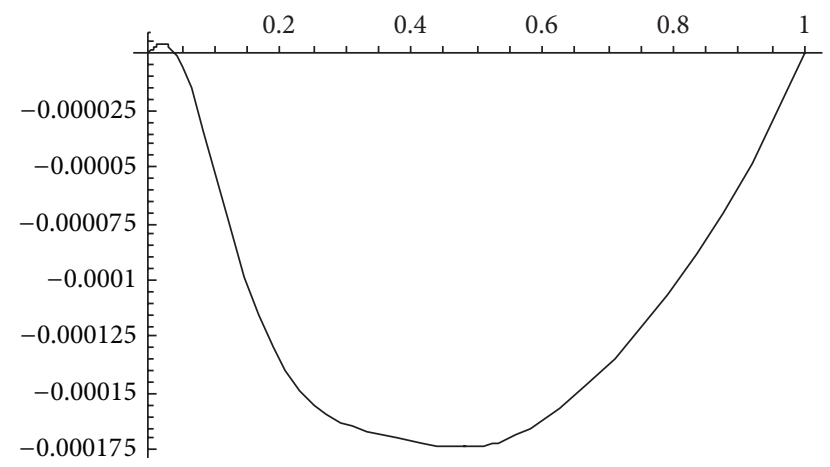

FIGURE 2: $y=0.2$, absolute error of the approximate solution $u(x)$, the exact solution $u \operatorname{true}(x), x \in[0,1]$.

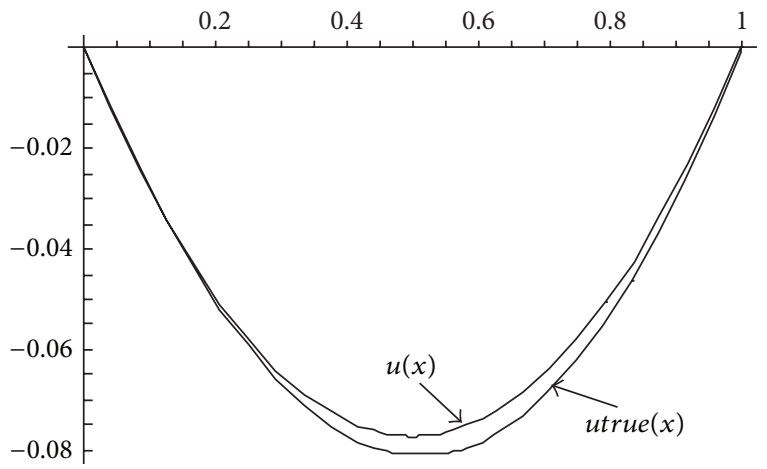

FIgURE 3: $y=0.9$, the approximate solution $u(x)$, the exact solution $\operatorname{utrue}(x), x \in[0,1]$.

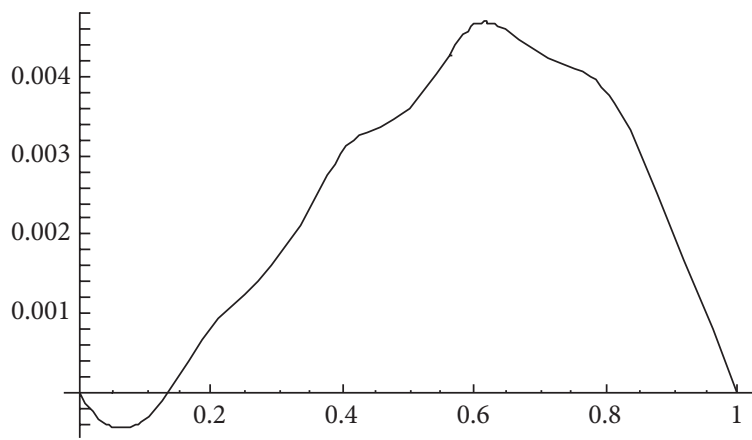

FIGURE 4: $y=0.9$, absolute error of the approximate solution $u(x)$, the exact solution $u \operatorname{true}(x), x \in[0,1]$.

\section{Conclusions}

Singular equation problems have wide application in engineering technology and increasingly penetrated into many fields of social science. In practice, a growing number of math problems and engineering problems to be converted into solving the singular equation. Therefore, the solution to mathematics and physics problem is of great significance. This paper discussed the solution to binary singular exact solution in the regeneration space. We give the exact solution expression in series. At the same time, yield its approximate solution through the study of the truncation of series and prove that the error between the approximation solution and exact solution is monotone decreasing and that $f_{n}(x)$ is the interpolation function of $f(x)$ accompanying the interpolation nodal. We also give some numerical examples to verify the accuracy of our method, and it turns out that our method is simple and effective.

\section{Conflict of Interests}

The authors declare that there is no conflict of interests regarding the publication of this paper.

\section{Acknowledgments}

The authors should say thanks to the support of Key Project of National Natural Science Foundation in China (nos. 71271069 and 91024028), National Key Technology R\&D Program of the Ministry of Science and Technology (no. 2012BAH81F03), Applied Technology R\&D Project in Heilongjiang Province (no. GC13D401), Humanities and Social Sciences Foundation of Chinese Ministry of Education (no. 10YJC860040), and National Soft Science Foundation in China (no. 2008GXS5D113).

\section{References}

[1] J. Mercer, "Functions of positive and negative type and their connection with the theory of integral equations," Philosophical Transactions of the Royal Society of London, vol. 1909, no. 209, pp. 415-446.

[2] S. Bochner, "Ein konvergenzsatz für mehrvariablige fouriersche Integrale," Mathematische Zeitschrift, vol. 34, no. 1, pp. 440-447, 1932.

[3] S. Zaremba, "Sur le calcul numerique des fonctions demandees dans le probleme De dirichlet et le probleme hydrodynamique," Bulletin International De 1'Academie De Sciences De Cracovie, vol. 196, pp. 125-195, 1908.

[4] N. Aronszajn, "Theory of reproducing kernels," Transactions of the American Mathematical Society, vol. 68, pp. 337-404, 1950.

[5] F. M. Larkin, "Optimal approximation in Hilbert space with reproducing kernel function," Mathematics of Computation, vol. 22, no. 4, pp. 911-921, 1970.

[6] M. M. Chawla and V. Kaul, "Optimal rules with polynomial precision for Hilbert spaces possessing reproducing kernel functions," Numerische Mathematik, vol. 22, no. 3, pp. 207-218, 1974.

[7] J. S. Chen, V. Kotta, H. Lu, D. Wang, D. Moldovan, and D. Wolf, "A variational formulation and a double-grid method for mesoscale modeling of stressed grain growth in polycrystalline materials," Computer Methods in Applied Mechanics and Engineering, vol. 193, no. 12-14, pp. 1277-1303, 2004.

[8] P. J. Angel, F. Pérez-Gonzalez, and J. Rattya, "Operatortheoretic differences between Hardy and Dirichlet-type spaces," http://arxiv.org/abs/1302.2422.

[9] B. Curgus and A. Dijksma, "On the reproducing kernel of a Pontryagin space of vector valued polynomials," Linear Algebra and Its Applications, vol. 436, no. 5, pp. 1312-1343, 2012.

[10] O. Chacaltana, J. Distler, and Y. Tachikawa, "Nilpotent orbits and codimension-2 defects of $6 \mathrm{~d} N=(2,0)$ theories," 
International Journal of Modern Physics A, vol. 28, no. 3-4, 54 pages, 2013.

[11] Y. Mu, J. Shen, and S. Yan, "Weakly-supervised hashing in kernel space," in Proceedings of the IEEE Computer Society Conference on Computer Vision and Pattern Recognition (CVPR '10), pp. 3344-3351, June 2010.

[12] M. Cui, "Two-dimensional reproducing kernel and surface interpolation," Journal of Computational Mathematics, vol. 4, no. 2, pp. 177-181, 1986.

[13] M. Cui and Z. Deng, "On the best operator of interpolation math," Numerical Sinica, vol. 8, no. 2, pp. 207-218, 1986.

[14] T. Várady, P. Salvi, and A. Rockwood, "Transfinite surface interpolation with interior control," Graphical Models, vol. 74, no. 6, pp. 311-320, 2012.

[15] Y. Kineri, M. Wang, H. Lin, and T. Maekawa, "B-spline surface fitting by iterative geometric interpolation/approximation algorithms," Computer Aided Design, vol. 44, no. 7, pp. 697-708, 2012.

[16] T. F. Jordan, Linear Operators For Quantum Mechanics, Dover, 2012.

[17] G. E. Fasshauer and Q. Ye, "Reproducing kernels of Sobolev spaces via a green kernel approach with differential operators and boundary operators," Advances in Computational Mathematics, vol. 38, no. 4, pp. 891-921, 2011.

[18] W. Boying, "Approximation regeneration of spatial function $W_{2}^{2}(D)$, , Natural Science Journal of Harbin Normal University, no. 3, pp. 14-24, 1995.

[19] B. Wu and Q.-L. Zhang, "The numerical methods for solving euler system of equations in reproducing kernel space," Journal of Computational Mathematics, vol. 19, no. 3, pp. 327-336, 2001.

[20] M. Mohammadi and R. Mokhtari, "Solving the generalized regularized long wave equation on the basis of a reproducing kernel space," Journal of Computational and Applied Mathematics, vol. 235, no. 14, pp. 4003-4014, 2011.

[21] O. A. Arqub, M. Al-Smadi, and N. Shawagfeh, "Solving Fredholm integro-differential equations using reproducing kernel Hilbert space method," Applied Mathematics and Computation, vol. 219, no. 17, pp. 8938-8948, 2013.

[22] S. Wang, F. Min, and W. Zhu, "Covering numbers in coveringbased rough sets," Lecture Notes in Computer Science, vol. 6743, pp. 72-78, 2011.

[23] S. Xiong, W. K. Liu, J. Cao, C. S. Li, J. M. C. Rodrigues, and P. A. F. Martins, "Simulation of bulk metal forming processes using the reproducing kernel particle method," Computers and Structures, vol. 83, no. 8-9, pp. 574-587, 2005. 


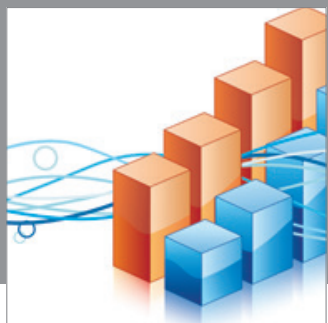

Advances in

Operations Research

mansans

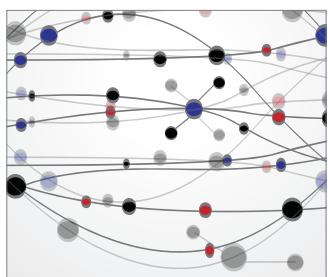

The Scientific World Journal
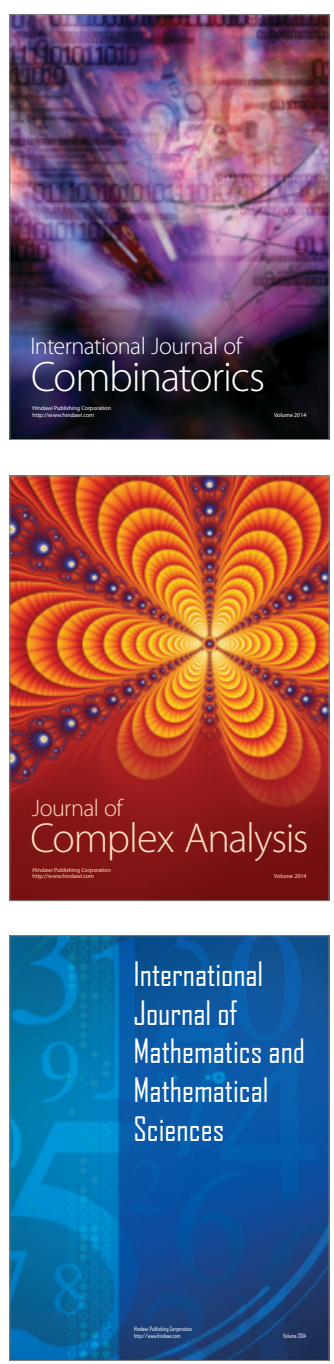
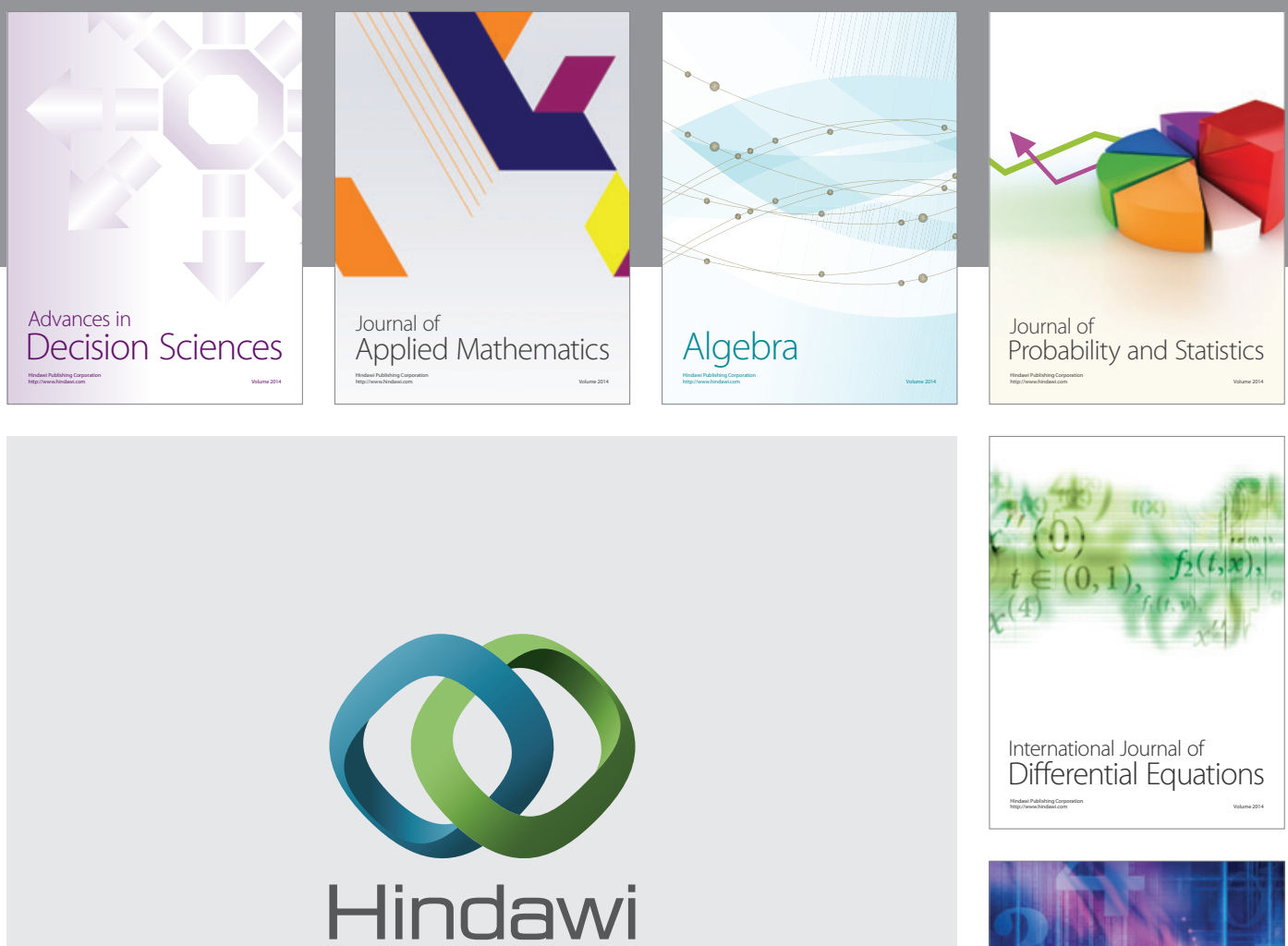

Submit your manuscripts at http://www.hindawi.com
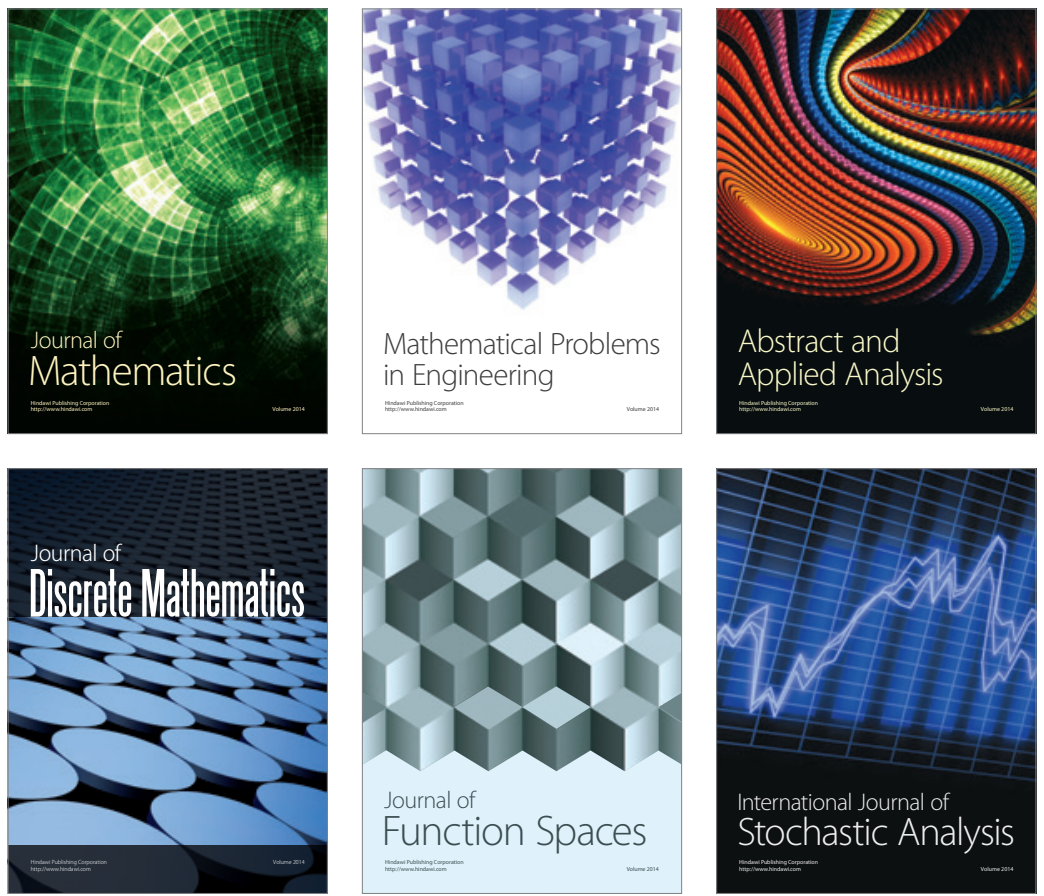

Journal of

Function Spaces

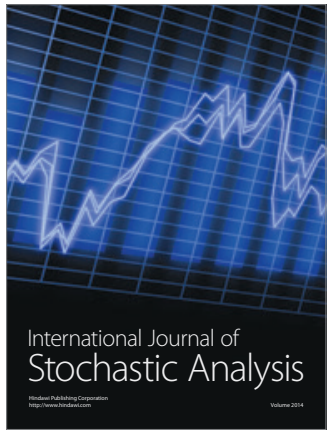

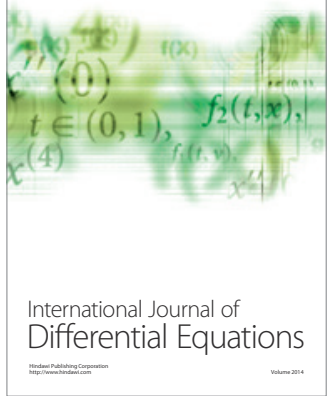
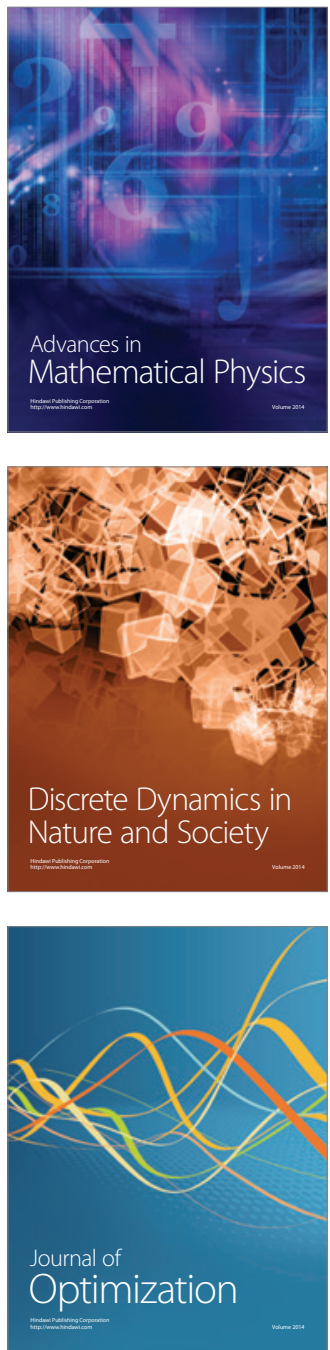